\title{
METABOLIC SYNDROME AND MUSCLE MASS
}

Authors:_Nilay Ergen ', Aslı Dogruk Unal' ${ }^{2}$, Ozlem Cigerli³, Hülya Parildar ${ }^{3}, O z l e m T a r c i n{ }^{2}$, Hatice Betül Ugur Altun' ${ }^{2}$,Nilgün GüvenerDemirag ${ }^{2}$

${ }^{1}$ Baskent University Istanbul Hospital, Department of Exercise Physiology ,Istanbul Turkey.

${ }^{2}$ Baskent University Istanbul Hospital, Department of Endocrinology and Metabolism, Istanbul, Turkey

${ }^{3}$ Baskent University Istanbul Hospital, Department of Family Medicine, Istanbul, Turkey

Background: Low skeletal muscle mass is an emerging risk factor for metabolic disorders. In this study, we aimed to evaluate the relationships between fat mass, muscle mass, muscle fat mass ratio, total fat mass, abdominal fat mass ratio by comparing the body compositions in obese women with and without metabolic syndrome Methods: Totally 89 obese women (mean \pm SD age $42,1 \pm 12$ years, BMI $36,5 \pm 4,2 \%$ ) with newly diagnosed metabolic syndrome (MS, $\mathrm{N}=64$, case group) and without metabolic syndrome (NMS, $\mathrm{N}=25$, control group) were included into study. Body composition was measured by bioelectrical impedance analysis (BIA). Metabolic syndrome was defined using the International Diabetes Federation 2005 diagnostic criteria.

Results: It was found that subjects with MS had $57,2 \pm 13,2 \mathrm{~kg}$ muscle mass, $41,2 \pm 8,9 \mathrm{~kg}$ of fat mass. Their muscle/fat ratio was $1,46 \pm 0,49$ and total fat/abdominal fat ratio was $1,99 \pm 0,25$. Subjects without MS had $53,5 \pm 8,4 \mathrm{~kg}$ of muscle mass, $39,2 \pm 9,3 \mathrm{~kg}$ of fat mass. Their muscle/fat ratio was $1,44 \pm 0,42$ and total fat/abdominal fat ratio was $1,96 \pm 0,37$ and there was no statistical significant difference $(p>0,05)$. On the other hand subjects who had MS and non-MS, fasting serum insulin and HOMA-IR values were $21,1 \pm 11,8 \mu \mathrm{U} / \mathrm{mL}$ and $11,3 \pm 5,5 ; 5,5 \pm 3,5 \mu \mathrm{U} / \mathrm{mL}$ and $2,7 \pm 1,5$, respectively $(p<0,0.1)$.

Conclusion: There is evidence that reduction in muscle mass and muscle/fat ratio is a risk factor for metabolic syndrome, but we did not find any significant reduction of muscle mass in subjects with MS in this study. This study should be redesigned in larger population to evaluate the meaning of these results. This may be explained by the anabolic effect of increased serum insulin levels in metabolic syndrome patients 